\title{
Development and Validation of a Partitioned Fluid-Structure Solver for Transonic Panel Flutter with Focus on Boundary Layer Effects
}

\author{
Marko Alder* \\ German Aerospace Center (DLR), Braunschweig, 38108, Germany
}

\begin{abstract}
A partitioned fluid-structure coupling code for transonic panel flutter has been developed and validated. The Reynolds-averaged Navier-Stokes equations are solved numerically by means of an implicit finite volume method to account for nonlinear aerodynamics, as there are shock waves and a viscous boundary layer at the panel surface. An implicit finite element formulation of the structural equations as well as a Galerkin solution of the von-Kármán plate equation are employed to solve elastic panel deformations with respect to geometric nonlinearities. A detailed validation process is presented in this paper for high subsonic and low supersonic Mach numbers. This comprises a discussion of available results from literature with the objective to propose a guideline for validation purposes of partitioned panel flutter solvers. Thereupon the code is used for studies on the impact of turbulent boundary layer characteristics on aeroelastic stability boundaries and postflutter. An evaluation of flutter modes and frequencies in the post-flutter domain as well as a discussion of the corresponding flow phenomena is presented.
\end{abstract}

\section{Nomenclature}
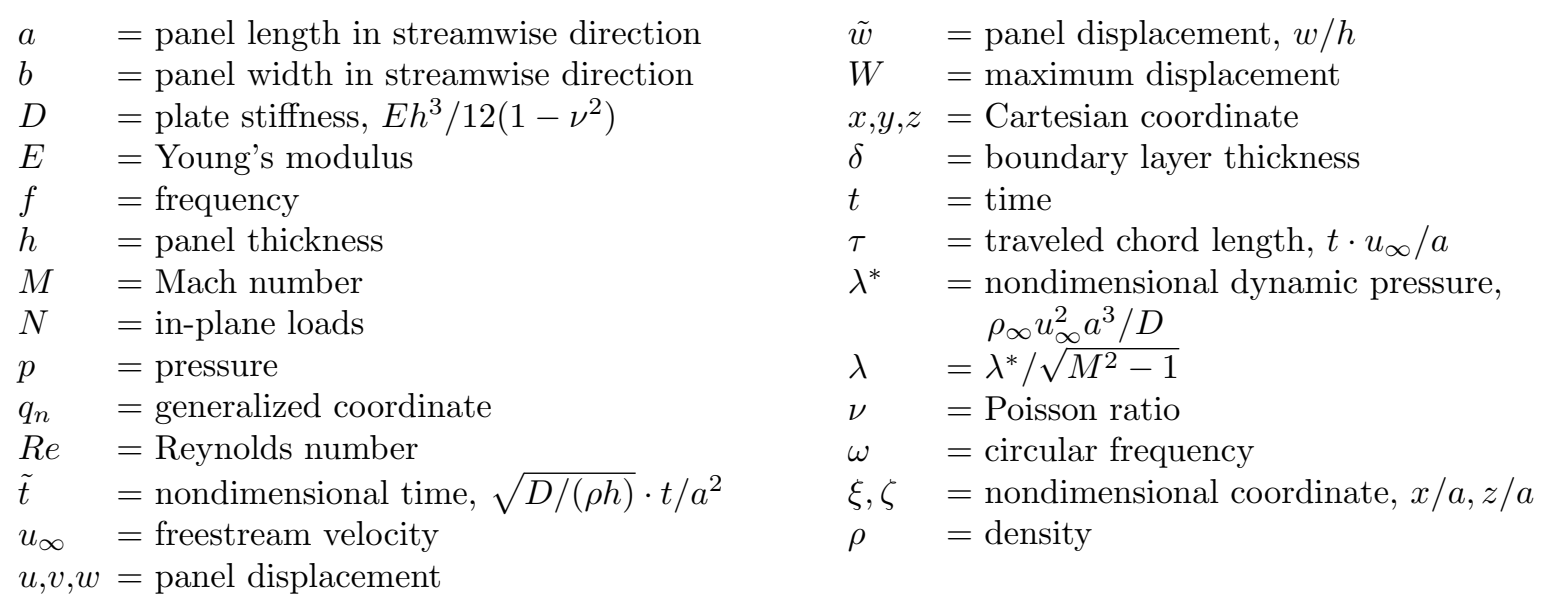

\section{Introduction}

ERODYnamic fairings of conventional launchers are sophisticated lightweight structures which are ex-

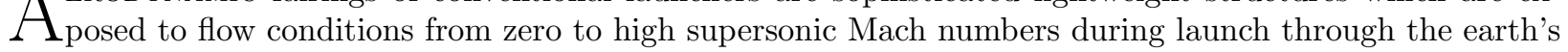
atmosphere. Coupling of the resulting aerodynamic loads with elastic and inertia loads can cause static or dynamic instabilities. The latter phenomenon is referred to as 'panel flutter' and was, inter alia, discovered on Atlas-Centaur or Saturn V rocket in the 1960s at NASA. ${ }^{1,2}$ There had been a considerable effort in these years to study the impact of numerous structural and aerodynamic parameters on aeroelastic stability. The

*Ph.D. Student, Institute of Aerodynamics and Flow Technology, Lilienthalplatz 7, AIAA member. 
majority of these studies comprises flat or cylindrical panels exposed to an airflow on the upper side and a cavity underneath. The panel edges are usually supported by a rigid frame. After the onset of divergence or flutter due to an increase in dynamic pressure the resulting amplitude is largely dominated by nonlinearities of the system. If deformations are in the order of the panel thickness, structural coupling between bending and stretching of the plate occurs. This in reverse limits the deformation. ${ }^{3}$ Such oscillations are referred to as limit cycle oscillation (LCO). ${ }^{4}$

Not only structural but also aerodynamic nonlinearities have an impact on the aeroelastic behavior of panels. Since the first are sufficiently understood for isotropic materials, the focus of this study will be on aerodynamic effects. Between the years 1950 and 1970 numerous theoretical studies applied essentially three levels of aerodynamic modeling. The simplest of these is (1) a quasi-steady relation between pressure and panel deflection, called 'piston' theory. ${ }^{5}$ Since this approximation is only valid for high supersonic Mach numbers $(M>\sqrt{2})$, a second approach is based on (2) unsteady linearized potential flow theory and considers effects of structural deformation at other points and at earlier times on the aerodynamic pressure. However, viscosity is not considered in isentropic flows and thus boundary layer effects at the panel surface are neglected. The third level of approximation is (3) the unsteady 'shear flow' theory developed by Dowell. ${ }^{3}$ This is based on the assumption that the mean velocity profile within a boundary layer impacts the aeroelastic behavior in particular. Applying a predefined velocity profile, i.e. a shear flow, in the vicinity of the structure, the balance equations of mass, momentum and energy in combination with the perfect gas formula can be combined to a single partial differential equation for the aerodynamic pressure. ${ }^{6}$ Back then this theory was the most suitable theoretical approach to account for a viscous boundary layer. The results have shown adequate agreement with experimental results by Muhlstein et al. ${ }^{7}$ for low supersonic Mach numbers. Both the shear flow theory and the experiments point out that for transonic flows the boundary layer significantly affects the aeroelastic stability boundary in a stabilizing manner. For a detailed overview of the various analytical and experimental results the publications of Dowell ${ }^{3,4,6}$ and Mei et al. ${ }^{8}$ are recommended.

With increasing computer capacity attention has been devoted to the development of sophisticated numerical methods, such as Finite Element Methods (FEM) and Computational Fluid Dynamics (CFD). Recent publications apply these methods to revive panel flutter studies with the focus on high subsonic and low supersonic Mach numbers. Davis and Bendiksen ${ }^{9}$ developed a solver for a tight coupling of the Euler equations and a finite element formulation of the nonlinear von-Kármán plate equation. Gordnier and Visbal ${ }^{10}$ account for a viscous boundary layer by coupling a solver for unsteady Navier-Stokes equations with a solution of the von-Kármán plate equation. A similar approach is realized by Hashimoto et al. ${ }^{11}$ aiming at a comparison of these new approaches with experimental results from Muhlstein et al. ${ }^{7}$ The authors observe an improved agreement compared to the shear flow theory as will be discussed in more detail in the context of code validation.

Multidisciplinary coupling of different solvers arises the need to consider numerical effects that come along with the implementation of coupling algorithms. Felippa et al. ${ }^{12}$ point out that in spite of the benefits compared to monolithic approaches numerical impacts on solutions have to be handled thoughtfully. These are in particular interpolation errors of state variables at the interface boundary of physical domains as well as numerical instabilities resulting from time lagging between structure and fluid. It is therefore in particular important to validate coupling algorithms with respect to these phenomena.

Whereas reduced order solutions (e.g. due to linearization) applied in numerous literature are aiming at a rapid determination of system eigenvalues to predict physical stability boundaries, transient partitioned solutions allow for a detailed analysis of the physical phenomenology of panel flutter even beyond flutter onset, in the following referred to as post-flutter. Thus, time accurate solutions by CFD methods are an important tool for detailed numerical studies of unsteady aerodynamics. However, whilst research has identified the importance of considering boundary layer effects, few numerical evaluations have been conducted focusing on the influence of turbulent boundary layer effects on the aeroelastic behavior of panels. Muhlstein et al. ${ }^{7}$ focus on flutter boundaries, while Gordnier and Visbal ${ }^{10}$ primarily study the impact of laminar boundary layers on aeroelastic stability and post-flutter. Although the latter conclude that laminar boundary layers induce significant damping effects at low supersonic Mach numbers, flow conditions of space launchers are predominantly turbulent.

A research cooperation between Airbus DS ${ }^{\mathrm{a}}$ and the German Aerospace Center (DLR) therefore aims at the development of numerical tools as well as an improvement of phenomenological understanding of panel flutter. The purpose of this paper is thus to investigate two subjects: First, based on the development of

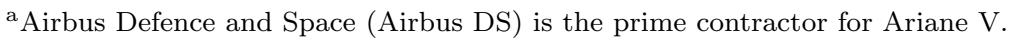


a partitioned fluid-structure coupling code, applicable literature sources as well as analytical methods are discussed and compared with own results whereby a reference guide for transonic panel flutter validation purposes will be proposed. Second, stability characteristics of a two-dimensional panel subjected to flow conditions from Mach 0.85 to 2.0 are investigated accounting for turbulent boundary layer effects. Within this context stability limits as well post-flutter characteristics are investigated.

For this purpose the following chapter is devoted to a description of applied numerical methods for the fluid, structure and coupling solution. In chapter three the boundary conditions are defined. Chapter four is devoted to a temporal and spatial resolution study, followed by the validation process and a study on the impact of viscous flow over a two-dimensional panel. Finally a conclusion will be drawn including an outlook on future activities.

\section{Computational Method}

\section{A. Aerodynamic Solver}

The DLR-TAU code, in the following called TAU, is used to solve the compressible three-dimensional NavierStokes and Euler equations in the integral form. Within the framework of a dual-cell approach a cell-centered finite volume method is applied. An upwind scheme (Roe's approximate Riemann solver) of second-order accuracy is used for spatial flux vector splitting.

The unsteady Navier-Stokes equations are integrated in time using an implicit dual time stepping approach by Jameson which is not restricted in the choice of the largest physical time step. This time step is chosen with respect to unsteady flow gradients as described later in the context of a grid and time convergence study. The convergence of each time step is controlled by a Cauchy convergence criteria with respect to lift and drag coefficients. A V-cycle on a multi-grid is performed for convergence acceleration.

Turbulent boundary layer effects are modeled by employing both the one-equation Spalart-Allmaras $(\mathrm{SA})^{13}$ and the two-equation $k-\omega$ model Menter SST ${ }^{14}$ in order to close the unsteady Reynold's Averaged Navier-Stokes (URANS) equations. Although the focus lies on the SA turbulence model, a comparison of these models highlights the impact of turbulence modeling on the aeroelastic stability. The flow solver itself is well validated with respect to a wide range of flow conditions, including transonic flows. ${ }^{15,16}$ Thus, a separate validation of the flow solution is neglected in this paper.

For reasons of comparability with simplified aerodynamic models the quasi steady, two-dimensional 'piston' theory will be applied as well. The pressure loading $p-p_{\infty}$ is obtained by:

$$
p-p_{\infty}=\frac{2 q}{\sqrt{M^{2}-1}}\left[\frac{\partial w}{\partial x}+\left(\frac{M^{2}-2}{M^{2}-1}\right) \frac{1}{u_{\infty}} \frac{\partial w}{\partial t}\right] .
$$

\section{B. Structural Dynamics Solver}

A Finite Element Method (FEM) based on the virtual work approach is employed to compute unsteady deformations of the structure within the Lagrangian formulation. The implicit nonlinear solution sequence SOL 400 by MSC Nastran is applied. Thin panels are discretized with shell elements based on the ReissnerMindlin plate theory to account for transverse shear deformations. ${ }^{17,18}$ An iterative adaption of the stiffness matrix allows to account for geometrical nonlinearities due to large structural displacements. A Newmark- $\beta$ scheme with adaptive time stepping is used for the time integration of the equations of motion.

In addition to the virtual work approach partial differential equations can be solved through a weighted residuals approach. Within this study the von Karman's large-deflection equation for two-dimensional plates is employed:

$$
D \frac{\partial^{4} w}{\partial x^{4}}-\left(N_{x}+N_{x 0}\right) \frac{\partial^{2} w}{\partial x^{2}}+\rho^{s} h \frac{\partial^{2} w}{\partial t^{2}}+\left(p-p_{\infty}\right)=\Delta p_{0} .
$$

In-plane load are composed of (externally) applied in-plane loads $N_{x 0}$ and nonlinear induced loading due to deflections of the panel:

$$
N_{x}=\frac{E h}{2 a} \int_{0}^{a}\left(\frac{\partial w}{\partial x}\right)^{2} d x .
$$

The aerodynamic loads $p-p_{\infty}$ represent the interface to the fluid solution. Substituting eq. (3) into (2) and 
applying appropriate nondimensional variables yields:

$$
\tilde{w}^{\prime \prime \prime \prime}-6\left(1-\nu^{2}\right)\left[\int_{0}^{1}\left(\tilde{w}^{\prime}\right)^{2} d \xi\right] \tilde{w}^{\prime \prime}-R_{x} \tilde{w}^{\prime \prime}+\frac{\partial^{2} \tilde{w}}{\partial \tilde{t}^{2}}+\frac{a^{4}}{D h}\left(p-p_{\infty}\right)=\Delta p_{0} .
$$

Equation (4) will be solved by a modal expansion and Galerkin's method. The weighting function defines the structural boundary condition. For a simply supported panel the deflection is thus defined as:

$$
\tilde{w}(\xi, \tilde{t})=\sum_{m} q_{m}(\tilde{t}) \sin (m \pi \xi) .
$$

After substituting eq. (5) into (4) the resulting equation will be multiplied by $\sin (s \pi \xi)$ and integrated over the panel length. Thus, eq. (4) can be reduced to a set of ordinary differential equations:

$$
\frac{d^{2} q_{s}}{d \tilde{t}^{2}}=-\left[(s \pi)^{2}+R_{x}(s \pi)^{2}+3(s \pi)^{2}\left(1-\nu^{2}\right) \sum_{r}(r \pi)^{2}\right] q_{s}-\frac{2 a^{4}}{D h} \int_{0}^{1} \Delta p(\xi) \sin (s \pi \xi) d \xi+\Delta p_{0} .
$$

The integral of the aerodynamic pressure loading along the panel length $\xi$ will be evaluated numerically using the Simpson's rule. After transforming the system of equations (6) into first order ordinary differential equations it will be integrated in time using the Fortran LSODA code. Dowell ${ }^{19}$ states that the system converges with 6 modes. Due to the today's computer capacity 15 modes will be considered in this study without major losses in computation time.

\section{Fluid-Structure Coupling}

\section{Coupling algorithm}

A non-overlapping spatial partitioning concept has been chosen to exchange state variables, i.e. loads $f$ and deformations $u$, between the fluid domain $\Omega^{f}$ and the structural domain $\Omega^{s}$. Figure 1 illustrates the coupling procedure. In advance a static flow field is computed. Applying a classical DirichletNeumann iteration the resulting loads $\left.f_{t_{0}}^{f}\right|_{\Gamma}$ at the interface boundary $\Gamma$ at $t_{0}$ are set as Neumann boundary condition of the structure $\left.f_{t_{0}}^{s}\right|_{\Gamma}$. Now a predictor step $n^{p}$ is conducted for the structural solution solving for the deformation $u_{t_{0}+\Delta t}^{s}$ at the end of the current load step of the length $\Delta t$. Afterwards this interface deformation $\left.u_{t_{0}+\Delta t}^{s}\right|_{\Gamma}$ is set as Dirichlet boundary condition for the fluid domain in order to compute the corresponding aerodynamic loads at $t_{0}+\Delta t$. Each predictor loop is followed by

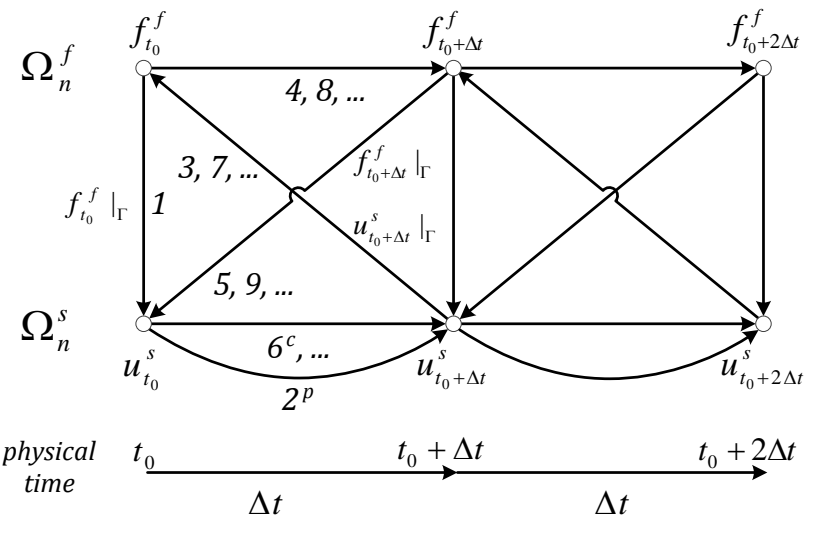

Fig. 1. Parallel staggered coupling scheme with subiterations. ${ }^{12}$ at least one corrector loop in terms of a subiteration strategy. This so-called tight coupling scheme synchronizes the fluid and the structure solution in time and is therefore of second-order accuracy. A study on the effect of lagging errors is presented by Gordnier and Visbal. ${ }^{10}$ They have shown that employing a loose coupling approach may cause numerical long-term instabilities of the coupled system. Usually between one and three corrector steps are sufficient to achieve a converged solution. The deviation $\varepsilon_{w}$ of structural deformations between two successive solutions serves as convergence criteria. It is defined by the total deformation vector $\left|\bar{w}\left(t_{n}\right)\right|$ :

$$
\varepsilon_{w}=\frac{\left|\bar{w}\left(t_{n+1}\right)\right|-\left|\bar{w}\left(t_{n}\right)\right|}{\left|\bar{w}\left(t_{n}\right)\right|} .
$$

\section{Interpolation}

Since the number of grid nodes usually differs at the interface between the structural and fluid domain an interpolation of state variables becomes necessary. In this study a scattered data interpolation based on 
radial basis functions $(\mathrm{RBF})$ is employed for aerodynamic loads, i.e. pressure and forces, as well as for structural deflections and mesh deformation. The basic assumption of this mathematical approach is that the extent to which a given point $i$ affects an arbitrary neighbor $j$ only depends on the Euclidean distance between both:

$$
f(x)=\sum_{i=1}^{N} \beta_{i} \phi\left(\left\|x-x_{i}\right\|\right)=h_{i} .
$$

Applying a volume-spline method a first degree polynomial is added to the $N$ centered radial basis functions in $\mathbb{R}^{3}$ :

$$
f(x)=\alpha_{1}+\alpha_{2} x_{i}+\alpha_{3} y_{i}+\alpha_{4} z_{i}+\sum_{i=1}^{N} \beta_{i} \phi\left(\varepsilon_{i}\right)
$$

with

$$
\phi\left(\varepsilon_{i}\right)=\varepsilon_{i}=\sqrt{\left(x-x_{i}\right)^{2}+\left(y-y_{i}\right)^{2}+\left(z-z_{i}\right)^{2}}
$$

and $\beta_{i}$ being the weighting factor of the basis function $\phi$. Equilibrium considerations of the sum of forces and moments yield:

$$
\sum_{i} \beta_{i}=0 ; \quad \sum_{i} \beta_{i} x_{i}=0 ; \quad \sum_{i} \beta_{i} y_{i}=0 ; \quad \sum_{i} \beta_{i} z_{i}=0 .
$$

Considering these assumptions and substituting (9) into (8) the $N+4$ unknowns can be determined by given point data $h_{i}$. For the sake of brevity the equations are not written out in detail since this is ordinary algebra. Nevertheless it is worth mentioning that up to this point all necessary coefficients for the calculation of the unknown variables are constant for each coordinate direction and only depend on the coordinate of the radial basis points. Thus, they must only be computed at the beginning of the coupled solution and then are stored as a $(N+4) \cdot(N+4)$ matrix to save computation time. Knowing the weighting factors of the basis points the unknown data $h_{j}$ can be determined by the transfer matrix $\overline{\bar{H}}$ :

$$
\overline{\bar{H}}=\left[\begin{array}{ll}
\overline{\bar{B}} & \overline{\bar{C}}
\end{array}\right]\left[\begin{array}{l}
\bar{\alpha} \\
\bar{\beta}
\end{array}\right]
$$

where

$$
\overline{\bar{B}}=\left[\begin{array}{cccc}
1 & x_{1} & y_{1} & z_{1} \\
1 & x_{2} & y_{2} & z_{2} \\
\vdots & \vdots & \vdots & \vdots \\
1 & x_{j} & y_{j} & z_{j}
\end{array}\right] ; \quad \overline{\bar{C}}=\left[\begin{array}{cccc}
\phi_{11} & \phi_{12} & \cdots & \phi_{1 i} \\
\phi_{21} & \phi_{22} & \cdots & \phi_{2 i} \\
\vdots & \vdots & \ddots & \vdots \\
\phi_{j 1} & \phi_{j 2} & \cdots & \phi_{j i}
\end{array}\right]
$$

Finally the interpolated values $h_{j}$ are obtained by:

$$
\overline{h_{j}}=\overline{\bar{H}} \cdot \overline{h_{i}} \text {. }
$$

Resulting mesh deformations are then linearly interpolated between two physical time steps $t_{n}$ and $t_{n+1}$ for the unsteady flow computation.

\section{Boundary conditions}

For validation purposes the computational domain in this study is orientated on the experimental work by Muhlstein et al. ${ }^{7}$ as well as the numerical studies by Hashimoto et al. ${ }^{11}$ and Gordnier and Visbal. ${ }^{10}$ Since the latter employ a slightly different panel geometry this will be accounted for by scaling the computational domain respectively. Figure 2 illustrates the 3D computational domain as well as the sectional plane that is used for representative $2 \mathrm{D}$ computations. According to the experimental work by Muhlstein et al. the length to width ratio of the $3 \mathrm{D}$ panel is 0.5 .

Deflections of the CSM edge nodes are not permitted $(u, v, w=0)$. Edge conditions are either clamped $(\partial w / \partial n=0)$ or pinned $\left(\partial^{2} w / \partial n^{2}=0\right)$. The panel is discretized by 101 nodes in the streamwise direction and 201 nodes in the spanwise direction. An initial perturbation of the inviscid fluid-structure system is achieved by defining an initial velocity boundary condition as:

$$
\frac{\partial w}{\partial t}(x, y)=c \cdot \sin ^{n}(\pi x / a) \cdot \cos ^{n}(\pi y / b) .
$$


The coefficient $\mathrm{c}$ is chosen reasonably small to avoid nonlinear effects and $n$ is either 1 or 2 for pinned or clamped edge conditions respectively. If the panel is exposed to a viscous boundary layer small pressure differences between the boundary layer and the cavity are sufficient to initiate a perturbation of the equilibrium state.

The fluid domain is defined as shown in figure 2. The lower boundaries define a solid adiabatic wall including the adiabatic elastic panel. A no-slip condition is set by the length $l_{x}$ so that a viscous boundary layer with a desired thickness can develop when solving the URANS equations. Values for the boundary layer thickness $\delta$ are related to the $99 \%$ freestream velocity above the center of the undeflected panel, unless noted otherwise by the index of $\delta$. Freestream condition is set at the inflow boundary and a first-order extrapolation at the outflow boundary. Since a freestream condition neglects flow gradients at the farfield this condition is not applicable at the left and right boundary of the computational domain which are exposed to the boundary layer. Therefore a slip condition is chosen. Freestream condition is set at the upper boundary which is far enough to prevent shock wave reflections. In the experimental studies by Muhlstein et al. ${ }^{7}$ the cavity pressure underneath the panel was controlled in order to obtain an initial zero differential pressure across the panel. Thus, in this study the average pressure over the panel is set as cavity pressure. The flow field is discretized by tetrahedral cells stretched in the normal wall direction and clustered near the wall. Furthermore the mesh is clustered at the leading edge of the no-slip wall as well

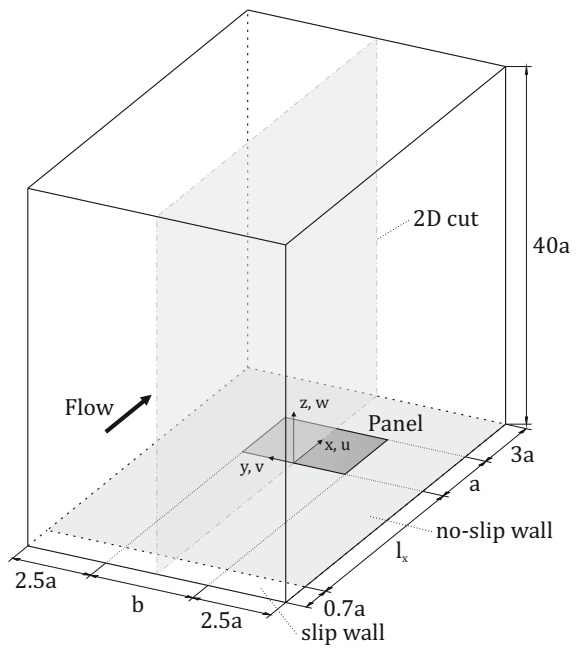

Fig. 2. Computational domain of the coupled fluid-structure system. as in the vicinity of the panel edges. The number of cells is varied with respect to a grid convergence study. The height of the first cell at the wall is reasonably small to achieve $y^{+} \approx 0.5$ across the panel.

\section{Results}

\section{A. Temporal and spatial resolution study}

It is highlighted by various authors that for the analysis of coupled fluid-structure problems the impact of temporal and spatial resolution on the aeroelastic behavior can be significant. ${ }^{10,11,20}$ Therefore, a variation of both parameters with respect to post-flutter and flutter boundaries is presented in the following. Grid convergence is proven by applying a coarse, medium and fine mesh resolution. The number of cells per unit of length in flow direction and in normal direction is $114 \times 71,229 \times 143$ and $457 \times 257$, respectively. The physical time is expressed in terms of traveled chord lengths $\tau$. Appropriate values of time step size $\Delta \tau$ are investigated with respect to different Mach numbers. The convergence study is based on viscous flutter with a turbulent boundary layer thickness of $\delta_{99} / a=0.05$. All cases are computed on $2 \mathrm{D}$ grids due to extensive requirements in computational time for $3 \mathrm{D}$ studies on high temporal and spatial resolution. Since the $2 \mathrm{D}$ grids are identical to the symmetry plane of the $3 \mathrm{D}$ grids it is supposed that normalized results of the convergence study are applicable to 3D grids as well.

A Richardson extrapolation is furthermore applied to the spatial and temporal resolution as described by Roache. ${ }^{21}$ Applying this method a Grid Convergence Index (GCI) is computed expressing a statistical error band on how the solution differs from its asymptotic value. Computing the relative error $|\varepsilon|$ of the coarser grid or temporal solution variables, the GCI is stated as:

$$
G C I_{\text {coarse }}=\frac{F_{s}|\varepsilon| r^{p}}{r^{p}-1}
$$

where $F_{s}$ is a factor of satisfactory. For the sake of reporting purposes $F_{s}=3$ is applied conservatively. Furthermore $r$ is the refinement ratio and $p$ the order of convergence. The latter is set to 1.75 to account for empirical constants within the boundary layer models.

Subject of a first study is a LCO under the condition of a nondimensionalized dynamic pressure of $\lambda^{*}=300$. An asymptotic behavior of the solution variables is observed, as expected, with decreasing time step size. Figure 3a illustrates the amplitude and frequency for a time step size varying from $\Delta \tau=0.4$ to 0.05 with a refinement factor of $r=2$. The results are normalized to its Richardson extrapolation. The 
respective GCI values are summarized in table 1 . It can be seen that a LCO amplitude is more sensitive to the temporal resolution than the associated frequencies. Furthermore the results deviate only very little on all three CFD grids used for the computations. A mesh resolution convergence study yields GCI values of less than one percent for both amplitude and frequency.

Furthermore the impact of the chosen time step size is investigated in terms of the critical dynamic pressure $\lambda_{c r i t}^{*}$. It was found that the sensitivity of the results to the temporal solution significantly depends on the Mach number and thereby the involved flutter modes. Figure 3b shows the stability boundary for Mach 1.2 as well as Mach 1.5. The results are again normalized to its Richardson extrapolation. Mach 1.5 requires a much smaller time step size in order to yield a converged solution. An individual convergence study, as shown exemplary for Mach 1.2 and 1.5, is conducted for other Mach numbers as well. In the following the solution is expected to be converged if the GCI value is less than three percent to account for an increase in flutter frequency with increasing $\lambda$.

Table 1. GCI values for the temporal resolution of a LCO for different spatial resolutions.

\begin{tabular}{lrrrrrr}
\hline \hline & \multicolumn{1}{c}{ coarse } & \multicolumn{3}{c}{ medium } & \multicolumn{2}{c}{ fine } \\
\cline { 2 - 7 }$G C I_{\Delta \tau}$ & $W$ & $\omega$ & $W$ & $\omega$ & $W$ & $\omega$ \\
\hline$G C I_{0.4-0.2}, \%$ & 13.48 & 10.06 & 13.81 & 10.19 & 12.80 & 10.09 \\
$G C I_{0.2-0.1}, \%$ & 4.05 & 3.24 & 3.40 & 2.71 & 3.74 & 3.05 \\
$G C I_{0.1-0.05}, \%$ & 0.97 & 0.70 & 1.31 & 1.05 & 1.08 & 0.78 \\
\hline \hline
\end{tabular}

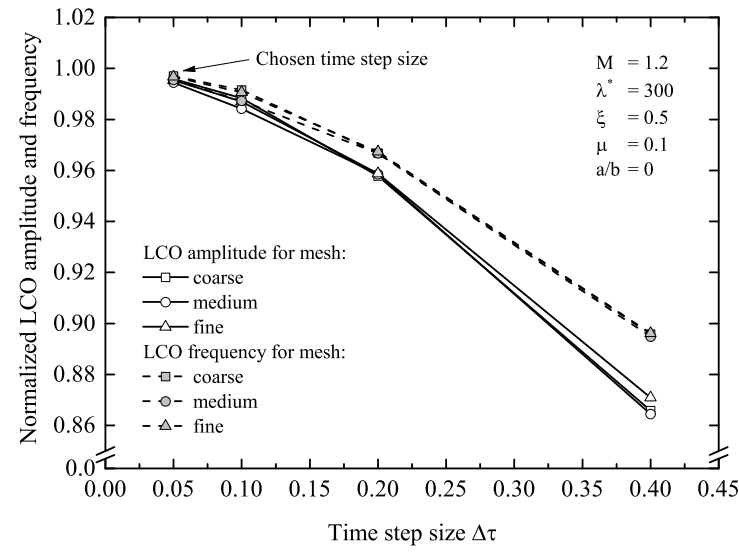

a) Limit cycle oscillation

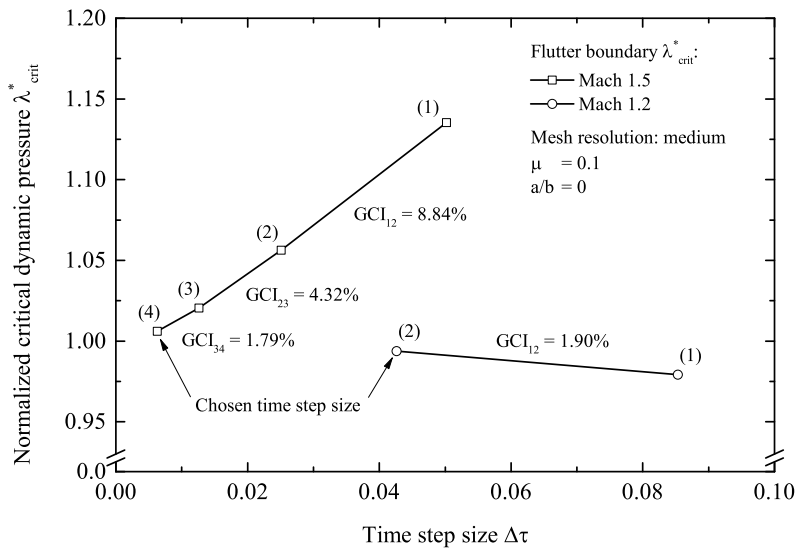

b) Flutter boundary

Fig. 3. Impact of time step and mesh resolution on post-flutter (LCO) and flutter boundary.

\section{B. Validation}

Although considering viscous effects is of major interest in the transonic Mach number regime the present study will beforehand primarily focus on inviscid panel flutter cases for validation purposes. Therewith results can be evaluated with respect to the numerical implementation of a coupling algorithm. Furthermore, both a solution of the von-Kármán-equation as well as a direct FEM formulation are employed. In the following an explicit comparison is neglected, since the results match exactly using the approaches described in chapter II.B. Selected examples of the validation process in this research project are presented for high subsonic, low supersonic and high supersonic ${ }^{\mathrm{b}}$ inviscid flows. In this context applicable literature is discussed.

\footnotetext{
b'In the context of this study the term 'high supersonic' is used for Mach numbers of approximately $M \geq 1.5$.
} 


\section{Subsonic flows}

A determination of stability boundaries for a pinned semi-infinite panel subjected to inviscid flow is conducted by Davis and Bendiksen. ${ }^{9}$ The dominant aeroelastic instability state of such a configuration under subsonic flow conditions is a static equilibrium state, i.e. aeroelastic buckling of a panel. In literature this effect is often referred to as static divergence. The critical dynamic pressure $\lambda_{\text {crit }}^{*}$ for the fluid-structure system to become unstable is decreasing with increasing Mach number. Figure 4 compares the results from Davis and Bendiksen with those computed within the current project. With a maximum deviation of $8.7 \%$ at Mach 0.85 the difference for subsonic Mach numbers is sufficiently small. For Mach 1.0 the difference is larger. However, by analyzing the potential flow theory, it can be shown analytically that the critical dynamic pressure of a semi-infinite panel $(a / b=0)$ is zero at $M=1$. Since an exact solution of the Euler equations should yield similar results, it is assumed that the reason for this deviation can be attributed to numerical discretiza-

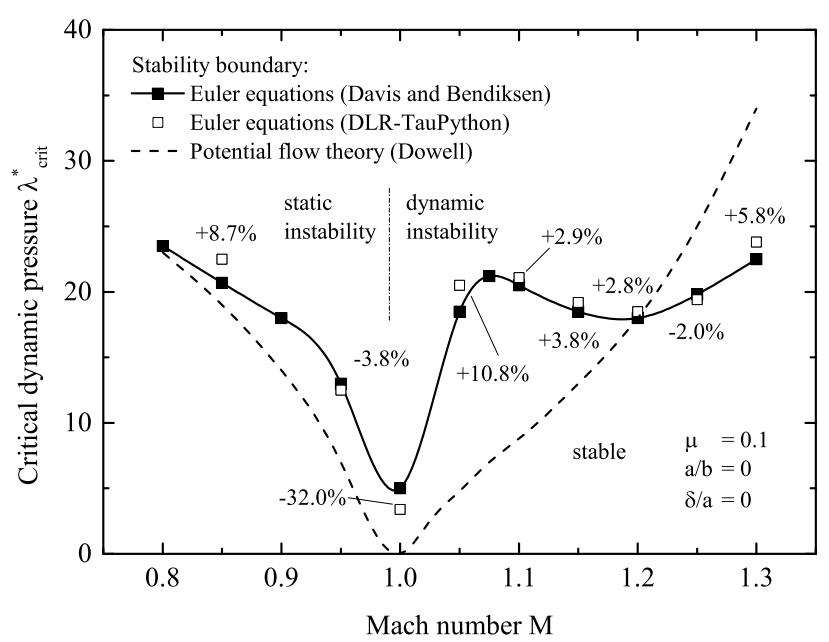

Fig. 4. Stability boundary in comparison with results from Davis and Bendiksen ${ }^{9}$ and Dowell. ${ }^{3}$ The deviations between potential flow theory and solutions of the Euler equations require further analysis.

tion. For panel aspect ratios of $a / b>0$ the critical dynamic pressure is larger than zero and thus the transonic dip will be much less pronounced. ${ }^{3}$ A more detailed analysis of the differences between solutions of the potential flow theory and Euler equations at high subsonic and low supersonic Mach numbers, as illustrated in Fig. 4, will be part of further studies.

Furthermore, Davis and Bendiksen ${ }^{9}$ as well as Gordnier and Visbal ${ }^{10}$ analyze such a configuration with respect to instability characteristics beyond flutter onset by solving unsteady Euler equations. Therewith the authors account for compressibility of the flow. A solution at Mach 0.95 is exemplarily chosen for the purpose of validation in order to highlight the dependence on the initial condition. As mentioned in chapter III an initial disturbance in the form of a sinusoidal velocity distribution perpendicular to the panel surface is applied. Depending on the direction of the velocity vector, i.e. a positive or negative initial velocity, the resulting static equilibrium state is either a positive or negative deflection. The results of the present code are compared with the results from Gordnier and Visbal in Fig. 5a in the form of the panel midpoint displacement. As this figure implies, the positive and negative deflections additionally differ in its absolute value. Furthermore, a remarkable effect occurs for $\lambda^{*}=2500$. Depending on the initial disturbance a negative static equilibrium state or a LCO may occur. The resulting oscillation is periodic, but involves higher frequencies due to a complex periodic shock movement above the panel surface. Figure $5 \mathrm{~b}$ illustrates transient displacements of surface points at $25 \%, 50 \%$ and $75 \%$ of the panel length. A detailed discussion of the corresponding flow field is given by the respective authors ${ }^{9,10}$ and is therefore not subject of the current work. At this point it is rather worth emphasizing that these results are well suited for validation purposes of subsonic Mach numbers since a coupling code can be validated with respect to solutions which depend on the applied initial conditions. Furthermore the impact of unsteady nonlinear flow effects on LCO oscillations gives confidence that the flow field is modeled with reasonable accuracy. Figure 5 highlights that positive and negative static instability as well as LCO oscillations match the results from Davis and Bendiksen. The difference in static equilibrium is within an error of one percent, while the difference in amplitude and frequency for the LCO is less than seven percent as summarized in table 2.

Table 2. Comparison of flutter amplitude and frequency (expressed as Strouhal number) for Mach 0.95 at $\lambda^{*}=2500$ with results from Gordnier and Visbal. ${ }^{10}$

\begin{tabular}{lrrr}
\hline \hline & Gordnier and Visbal & DLR-TauPython & Difference, $\%$ \\
\hline $\mathrm{W} / \mathrm{h}$ & 7.38 & 7.0 & -5.2 \\
$\mathrm{St}$ & 0.031 & 0.029 & -6.5 \\
\hline \hline
\end{tabular}




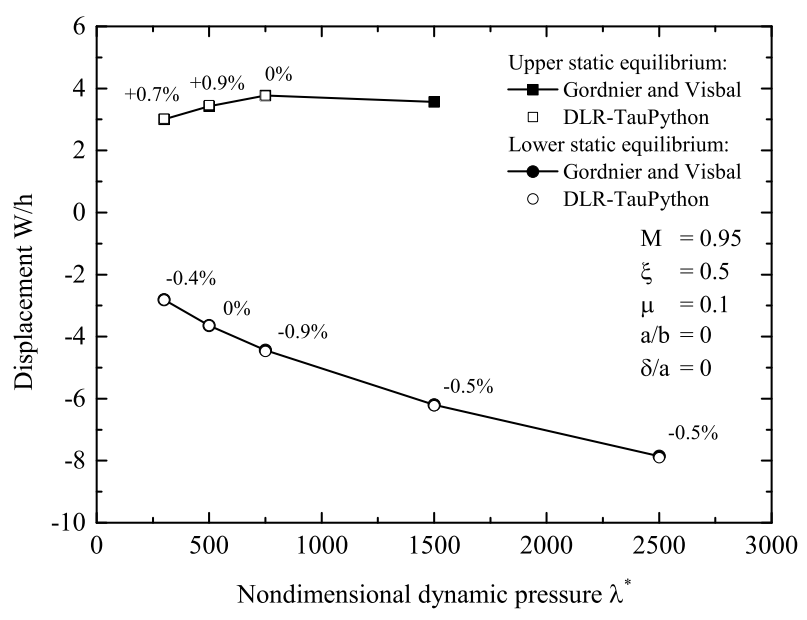

a) Static deflection at $\xi=0.5$

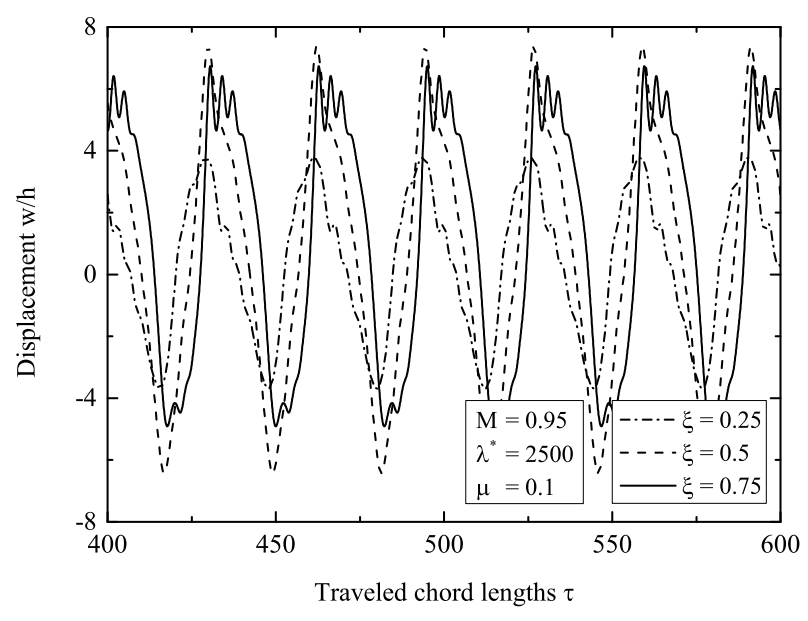

b) Dynamic deflection during LCO

Fig. 5. Panel divergence and LCO at Mach 0.95 for subsonic flows results from Gordnier and Visbal. ${ }^{10}$

In addition to pinned and clamped boundary conditions on all four edges, LCO at subsonic flows may under certain circumstances occur for other boundary conditions, e.g. for a free trailing edge. This shall be remarked since aerodynamic fairings of launchers are sometimes mounted in such way. Dugundji, et. al ${ }^{22}$ present analytical as well as experimental results concerning this topic. Since the focus of this paper is on the impact of boundary layer effects, the following studies will only imply more generic models with fixed boundary conditions as described in chapter III.

\section{Low supersonic inviscid flows}

Once the dynamic pressure exceeds a criticial value $\lambda_{c r i t}^{*}$, thin panels subjected to low supersonic flow conditions are characterized by dynamic instabilities, i.e. flutter. Figure 4 shows a comparison of results from Davis and Bendiksen ${ }^{9}$ with own results solving unsteady Euler equations as mentioned in the previous chapter. Between Mach 1.05 and 1.3 an increase of the critical dynamic pressure is followed by a local minimum at Mach 1.2. In literature the stability limit is often considered as a continuous curve ranging from subsonic to supersonic Mach numbers. ${ }^{3,9}$ Thereby it should be noted that for inviscid flow both static and dynamic instability may exist simultaneously for Mach 1.0. As analyzed by Davis and Bendiksen dynamic instability occurs at dynamic pressures significantly larger than those for static instability. ${ }^{9}$ For the sake of validation the results computed with the current coupling code show sufficient agreement with those computed by the latter authors.

In addition to the stability limit the dynamic behavior beyond flutter onset is studied for Mach 1.2 and 1.414. Figure 7 compares respective LCOs

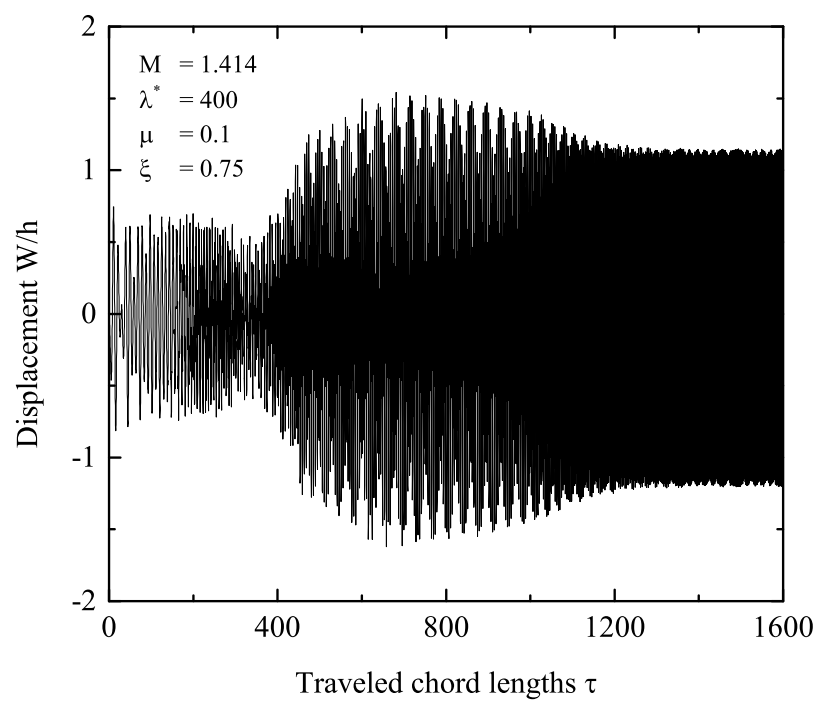

Fig. 6. Transient mode change at Mach 1.414 after an initial excitation in form of a mode 1 velocity distribution. This effect has also been observed by Gordnier and Visbal. ${ }^{10}$ in terms of flutter amplitude and frequency. Two literature sources are employed for validation. Dowell is using a linear potential flow theory in order to solve for unsteady aerodynamic loads. ${ }^{23}$ Furthermore, Gordnier and Visbal apply unsteady Euler equations as noted before. An increase in dynamic pressure results in a rapid increase of a periodic panel deflection in the form of the first structural mode shape. In 
conjunction with this shock waves and expansion fans alternately form at the leading and trailing edge of the panel over a LCO period at Mach 1.2. At Mach 1.414 an effect is observed, which is important to consider when validating coupling codes. Figure 6 shows the transient deflection of a point at $75 \%$ of the panel length. While the initial oscillation in the form of the first structural mode is damped a higher mode is excited simultaneously. After a time-intensive computation the amplitude converges to an asymptotic value. Gordnier and Visbal observe a dominant mode in the form of the third natural mode shape of the structure. The authors provide a detailed discussion of the unsteady flow characteristics ${ }^{10}$ as well as results in form amplitudes and frequencies for Mach numbers 1.2, 1.414 and 1.8 and both pinned and clamped structural boundary conditions. Thus, this source is well suited for validation of coupling codes with focus on high fidelity modeling of unsteady aerodynamics. With respect to the current coupling code the differences to the results from Gordnier and Visbal are shown in Fig. 7a for amplitude and Fig. 7b for the respective flutter frequencies. The maximum deviation for both amplitude and frequency is smaller than $5 \%$ and less than $1 \%$ for Mach 1.414. Therefore it can be stated that the coupling code is capable to predict LCO correctly even if the initial disturbance differs from the final oscillation mode.

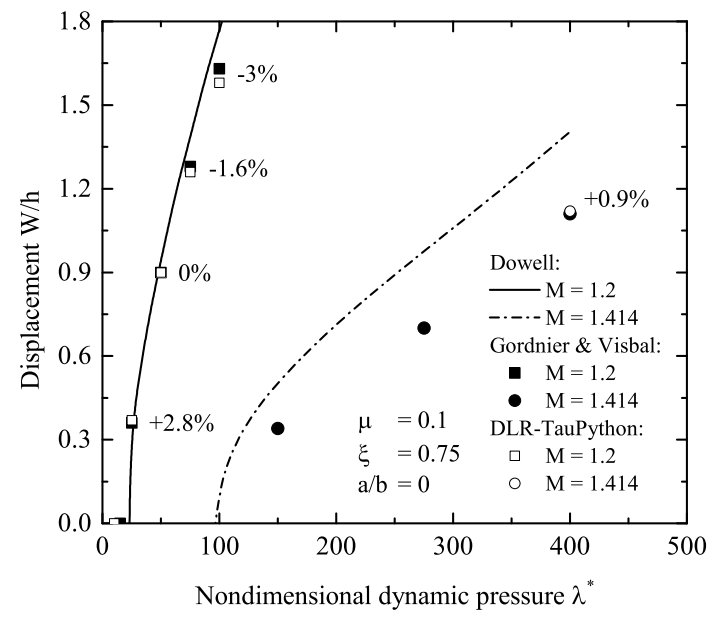

a) Amplitude

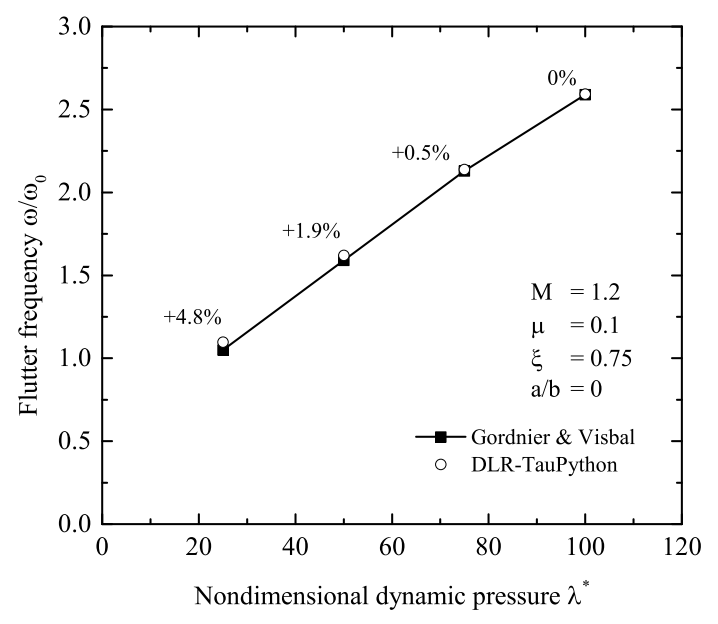

b) Frequency

Fig. 7. LCO amplitudes and frequencies at Mach 1.2 and Mach 1.414 in comparison with results from Gordnier and Visbal $^{10}$ (Euler equations) and Dowell ${ }^{24}$ (potential flow theory) for a pinned semi-finite panel.

\section{Low supersonic viscous flows}

At this point two additional parameters shall be considered for low supersonic flows. First, the width of the panel and fluid domain will be finite. The respective dimensions are defined in chapter III. Furthermore aerodynamic nonlinearities in terms of viscosity of the flow are taken into account. Various authors highlight that a viscous fluid boundary layer cannot be neglected for low supersonic flows. ${ }^{6,7,11}$ Muhlstein et al. ${ }^{7}$ conduct experimental stability analysis from Mach 1.05 to 1.4 for a clamped panel. Hashimoto et al. ${ }^{11}$ has shown that solving URANS equations in conjunction with a Spalart-Allmaras turbulence model yields improved results compared to Dowell's shear layer extension of the potential flow theory. ${ }^{6}$ The results are summarized in Fig. 8 for a boundary layer thickness of $\delta_{98} / a=0.1$.

This literature serves as an excellent source for validation of panel flutter coupling codes. Moreover Muhlstein et al. discuss correlations between various boundary layer characteristics, e.g. a varying thickness, and the aeroelastic response of the system. ${ }^{7,25}$ Nevertheless, experimental results must also be considered critically in terms of an error band. This is performed by Muhlstein conducting a forced-vibration technique for a more accurate determination of stability limits. He concludes that the determination of flutter boundaries using conventional methods, i.e. free flutter without actuators, is quite accurate in the vicinity of Mach 1.1. However, at higher Mach numbers, the conventional methods yield a flutter boundary approximately $10 \%$ below the boundary obtained with an accurate forced-vibration technique. ${ }^{26}$ Having this in mind the numerical and experimental results agree well as shown in Fig. 8. The difference of the current coupling code is within 10\% for Mach 1.4 and $13 \%$ for Mach 1.1. Nevertheless the results 
match those by Hashimoto. Thus, the conservative prediction of the stability boundary at Mach 1.1 is assumed to result from turbulence modeling rather than the implementation of the coupling algorithm.

\section{High supersonic flows}

For Mach numbers of $M>\sqrt{2}$ a decreasing shock angle allows for the assumption that pressure gradients are large perpendicular to the panel surface compared to the gradients in flow direction. Thus, the quasi-steady 'piston' theory becomes applicable. Because of the simplicity of this aerodynamic model numerous literature sources are available covering high supersonic panel flutter analysis. For the purpose of validation equation (1) is substituted into equation (4) and then numerically solved for the flutter boundary. Furthermore Dowells solutions of the linearized potential flow theory ${ }^{23}$ as well as Euler solutions from Davis and Bendiksen ${ }^{9}$ are employed. Figure 9a compares these results in terms of the critical dynamic pressure $\lambda_{c r i t}^{*}$. The largest difference is found for Mach 1.5. The deviation can be attributed to the high frequency flutter phenomenon as described for Mach 1.414 (Fig. 6). Transient results are shown and discussed in more detail by Davis and Bendiksen. ${ }^{9}$ For Mach numbers of 1.6

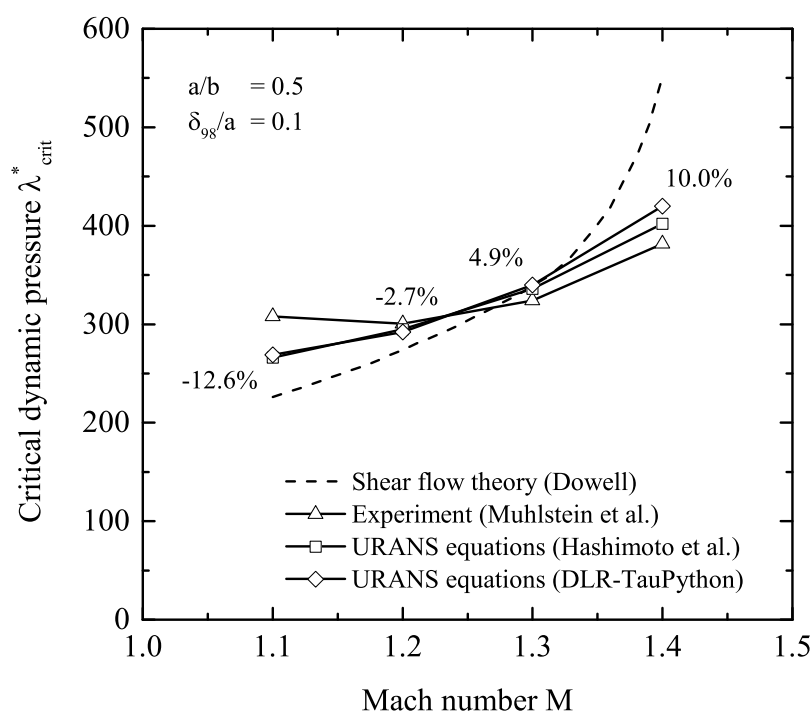

Fig. 8. Viscous validation of three dimensional panel flutter based on Muhlstein et al., ${ }^{7}$ Dowell $^{6}$ and Hashimoto. $^{11}$ The error indicators quantify the discrepancy to experimental results expressed as a percentage. and larger the results approach each other.

The post-flutter behavior is exemplarily validated for Mach 2. As also published by Dowell ${ }^{24}$ for the same boundary conditions ${ }^{\mathrm{c}}$, amplitude and frequency are computed for $\lambda=500\left(\lambda^{*}=866\right)$. Figure 9b compares the flutter mode shape obtained by solving piston theory and Euler equations. Although the lower deflection differs a little bit, the deformation shows a coupling of two structural natural modes, which is represented well by both theories. A direct comparison of the results yields a difference of $2.7 \%$ in amplitude and $13.4 \%$ in frequency (see table 3).

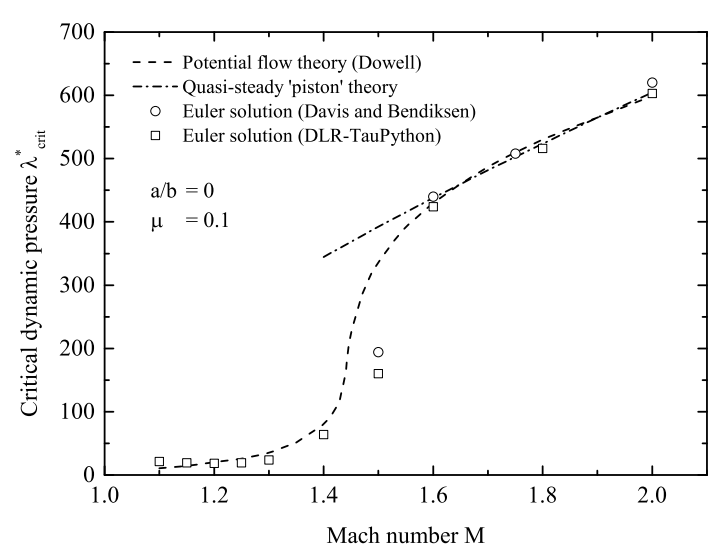

a) Stability boundary

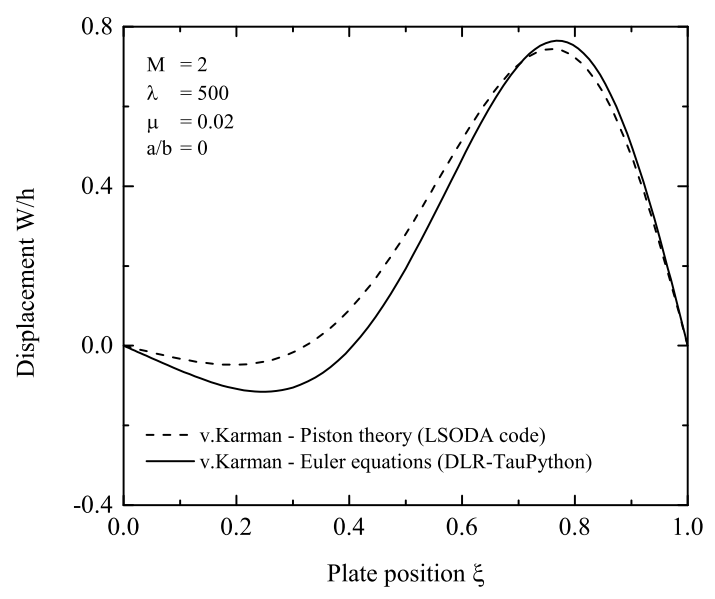

b) LCO at Mach 2

Fig. 9. Stability boundary and post-flutter mode shape of a simply supported panel at supersonic flow.

\footnotetext{
${ }^{\mathrm{c}}$ As mentioned before a superposition of 15 modes instead of 6 modes is used within this study compared to the computations by Dowell.
} 
Table 3. Comparison of flutter amplitude and frequency for Mach 2.0 at $\lambda=500$.

\begin{tabular}{lrrr}
\hline \hline & Quasi-steady flow theory & DLR-TauPython & Difference, $\%$ \\
\hline $\mathrm{W} / \mathrm{h}$ & 0.74 & 0.76 & +2.7 \\
$\omega / \omega_{0}$ & 4.06 & 4.60 & +13.4 \\
\hline \hline
\end{tabular}

\section{Impact of a turbulent boundary layer on stability and post-flutter behavior}

After the successful validation of the coupling code it will now be used to study the impact of viscous boundary layer effects on the stability of the fluid-structure system. A parameter study is aiming at a determination of the aeroelastic behavior of a flat panel subjected to turbulent boundary layers. As part of this paper the following questions will be subjected:

- How does a turbulent boundary layer affect the stability boundary of a 2D simply supported panel?

- Are 2D results qualitatively comparable to 3D panel flutter results?

- How does a turbulent boundary layer affect the post-flutter behavior at low supersonic flows?

Dimensions, boundary conditions and discretization methods as described in the previous chapters (refer to III) are applied with respect to two-dimensional computations of a panel, i.e. $a / b=0$, pinned at both edges. A mass ratio of $\mu=0.1$ will be maintained constant over a Mach number range from 0.85 to 2.0. All results are computed for a Reynolds number of $R e=7 \cdot 10^{6}$ with respect to the panel length $a$. In order to alter the dimensionless dynamic pressure $\lambda^{*}$, the panel stiffness is varied. Unless noted otherwise the results are computed for a boundary layer thickness of $\delta_{99} / a=0.05$ at $50 \%$ of the panel length. Since the validation process has shown good agreement between theory and experiment using the Spalart-Allmaras turbulence model, the following study is as well based on this model.

\section{Stability boundary}

The first subject of investigation is the transition from stable to unstable behavior of the panel. As discussed in chapter IV.B instability can be characterized as static or dynamic instability. Figure 10a compares the critical dynamic pressure $\lambda_{c r i t}^{*}$ for inviscid and viscous flows. For subsonic Mach numbers including Mach 1.0 static instability, i.e. aeroelastic buckling, was found for inviscid as well as viscous computations. It has to be noted that an exact determination of the stability limit is hardly possible if turbulent boundary layers are taken into account. With increasing dynamic pressure the deflection amplitude begins to grow linearly. At an amplitude of approximately 3 to $5 \%$ of the panel thickness an exponential growths sets in. Therefore an extrapolation of the latter for the dynamic pressure for zero deflection is assumed as stability boundary. Based on this consideration the turbulent boundary layer shows a damping character, i.e. the critical dynamic pressure is slightly increasing. While the absolute value for onset of static divergence rapidly decreases with increasing Mach number, the relative difference between inviscid and viscous results, expressed by equation (17), is less than $22 \%$ as illustrated in Fig. 11.

$$
\Delta(\text { viscous }, \text { inviscid })=\frac{\lambda_{\text {visc. }}^{*}-\lambda_{\text {invisc. }}^{*}}{\lambda_{\text {invisc. }}^{*}} \cdot 100 \%
$$

A damping effect of the turbulent boundary layer can also be observed for low supersonic Mach numbers. In contrast to subsonic flow conditions the instability can now be characterized as dynamic, i.e. the system begins to flutter. In connection with this result it must be noted that a numerical study from Dowell, applying the shear flow theory, leads to the conclusion that instability can be static if the boundary layer thicknesses is sufficiently large. ${ }^{28}$ The thickness required to suppress dynamic instability increases with increasing Mach number. Applying a boundary layer thickness of $5 \%$ of the panel length, flutter already occurs at Mach 1.05. Thus, the transition from static to dynamic instability in the vicinity of critical dynamic pressure is assumed to be in between Mach 1.0 and 1.05. The difference in terms of the critical dynamic pressure reaches a maximum of $47 \%$ at Mach 1.2. Fig. 10b illustrates the associated flutter frequencies, normalized to the panel natural frequency, and mode shapes. Regardless of the boundary layer the flutter mode shape is similar 


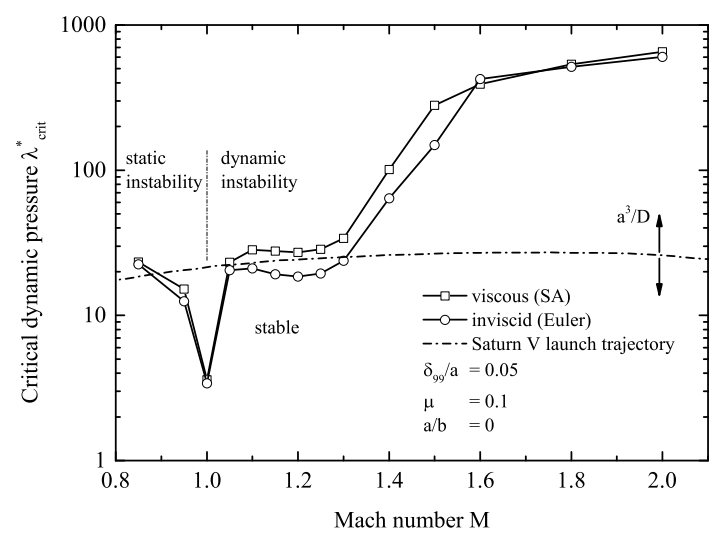

a) Stability boundary

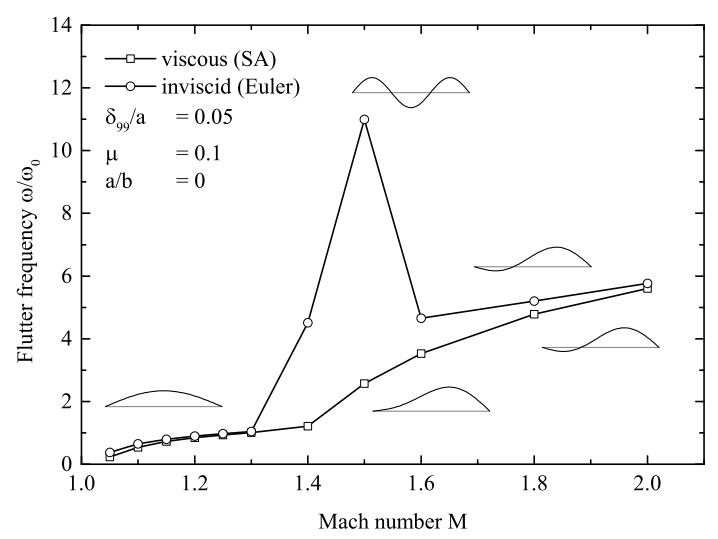

b) Flutter frequency

Fig. 10. Comparison of inviscid and viscous computations in terms of stability boundaries and corresponding flutter frequencies for varying Mach numbers. The considerable decrease in critical dynamic pressure in the vicinity of Mach 1 would be much less pronounced for three-dimensional panel $(a / b>0) .^{3}$

to the first natural panel mode shape with flutter frequencies equal or lower than the natural frequency of the structure. In literature this phenomenon is referred to as 'single mode' flutter ${ }^{4}$ which is, in contrast to typical mode coalescence at flutter onset, characterized by a negative aerodynamic damping of the flow responding to a first mode oscillation. Approaching Mach 1.3 the flutter frequencies of viscous and inviscid computations approach each other. Fig. 11 highlights that flutter frequencies are reduced when accounting for a viscous boundary layer. However, this effect is small for Mach numbers lower or equal than 1.3.

A further increase in Mach number shows significant deviations between inviscid and viscous computations. The difference in critical dynamic pressure is increasing until a maximum of almost $90 \%$ is reached for Mach 1.6. An explanation can be drawn from analyzing the corresponding flutter modes. From Figure 10b it can be concluded that by neglecting boundary layer effects the flutter mode is of the third panel natural mode. This involves higher flutter frequencies. Taking a turbulent boundary layer into account a damping effect on the high frequency flutter is observed and the panel mode shape is dominated by the first panel natural mode. However, an analysis of the corresponding frequency spectra, i.e. FFT analysis, indicates an increased influence of higher frequencies compared to lower Mach numbers. Thus, particular attention must be paid to a sufficient temporal resolution as discussed in chapter IV.A.

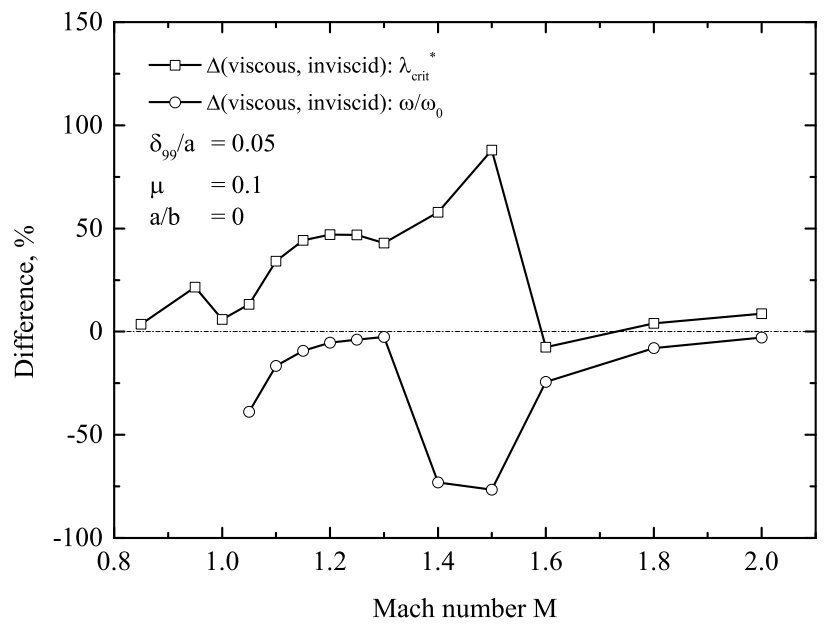

Fig. 11. Difference between inviscid and viscous panel flutter with respect to stability boundary and flutter frequency.

At high supersonic Mach numbers, i.e. $M \geq 1.6$ dynamic instabilities can be characterized by a distinctive coupling of the first and second panel natural mode for both inviscid and viscous computations. The impact of the turbulent boundary layer on critical dynamic pressure and flutter frequency is decreasing with increasing Mach numbers. In the region of $M=1.6$ a destabilizing effect of the boundary layer on the critical dynamic pressure can be observed.

With respect to the application of space launchers lower Mach numbers are more critical as shown in Figure 10a. The dynamic pressure a launcher is exposed to along its launch trajectory is illustrated for the case of a Saturn V rocket. ${ }^{27}$ It qualitatively agrees well with today's launch systems of comparable size, e.g. Ariane V. Accounting for turbulent boundary layers allows for reduced panel stiffness or larger panel 
lengths. Thus, in the case of the boundary conditions applied within this study, $M=1.2$ can be stated as a critical design point.

Since the stabilizing effect of a turbulent boundary layer in terms of single mode flutter is the largest in the vicinity of $M=1.2$, it will now be chosen for further studies. Figure 12 illustrates the dependence of the critical dynamic pressure on the boundary layer thickness. The two-equation turbulence model Menter SST is as well applied for a normalized boundary layer thickness of $5 \%$, yielding a similar flutter boundary as by using the SA model. Up to $\delta_{99} / a=0.1$ a linear dependence is observed. This is in agreement with experimental results from Muhlstein at al. ${ }^{7}$ Inviscid computations, however, show a slightly higher critical dynamic pressure as could be expected by an extrapolation of the viscous flow results. Applying the shear flow theory, the linear relationship between boundary layer thickness and critical dynamic pressure for flutter onset is furthermore confirmed by Dowell for two different aspect ratios $(a / b=0.5$ and $a / b=2.0)$ and clamped boundary conditions. ${ }^{28}$

\section{Post-flutter characteristics}

An increase in dynamic pressure beyond the stability boundary generally involves an increase in ampli-

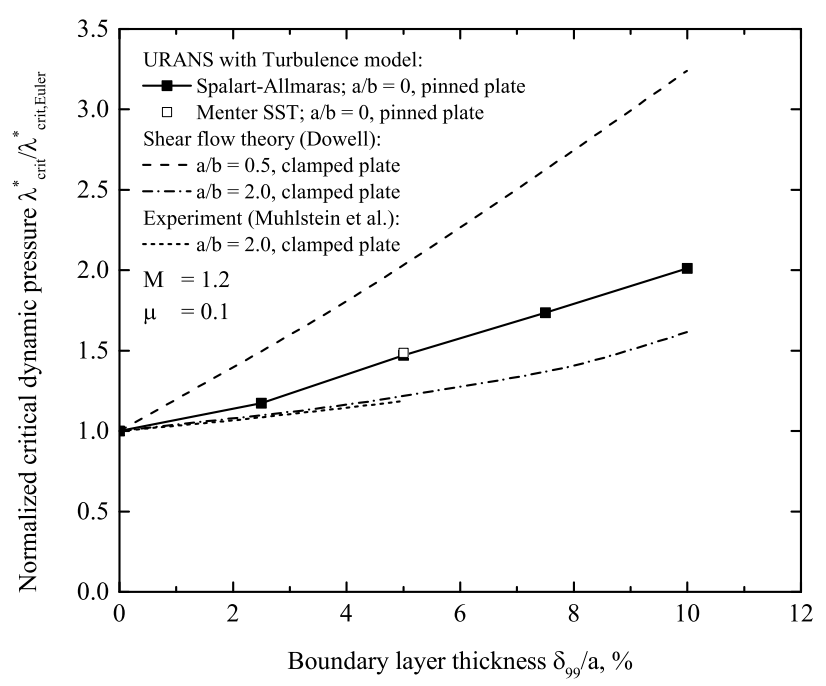

Fig. 12. Flutter boundary of a flat plate subjected to Mach 1.2 flow conditions with varying boundary layer thickness. While the slope of the curves depend on the aspect ratio and boundary conditions of the plate, experimental results from Muhlstein et al. ${ }^{7}$ as well as analytical results from Dowell ${ }^{28}$ confirm the linear relationship for moderate boundary layer thicknesses.

tude and frequency. For the sake of brevity the focus within this paper is again on Mach 1.2. Figure 13 compares normalized maximum amplitudes $W_{\max } / h$ (Fig. 13a) and frequencies $\omega / \omega_{0}$ (Fig. 13b) of inviscid and viscous computations for various dimensionless dynamic pressures $\lambda^{*}$. The deviation $\Delta$ (inviscid, viscous) between the results is plotted in the respective diagram. It can be seen that the turbulent boundary layer reduces the resulting amplitude and frequency as the dynamic pressure is beyond flutter onset. However, there exists a point at which the inviscid solution suddenly alters. Because of a transition from mode 1 to mode 2 flutter the associated amplitude is decreasing while the frequency is significantly increasing. By taking the boundary layer into consideration this mode change is suppressed. The non-monotonous behavior just before the transition and the dependence of the LCO stability on numerical boundary conditions requires further detailed analysis.

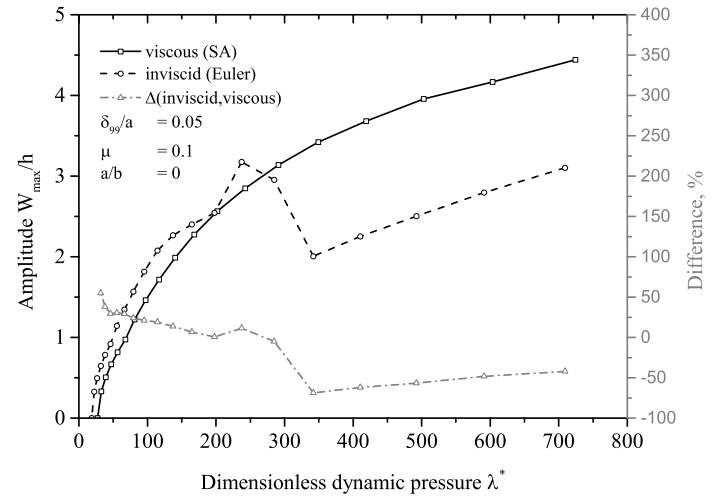

a)

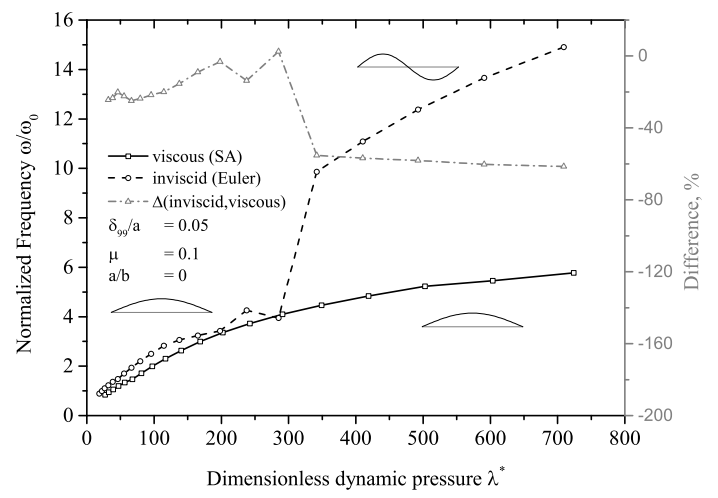

b)

Fig. 13. Normalized flutter amplitude and frequency with respect to dynamic pressure.

For a better understanding of the physical phenomenology the flow field above the flexible panel is plotted in Fig. 14 in terms of the local pressure coefficient $c_{p}$. The maximum deflection at similar $\lambda^{*}$ before and 
after the flutter mode change is shown. Low dynamic pressures are represented by Fig. 14a and Fig. 14b. Both inviscid and viscous computations show a deformation of the panel in the form of its first natural mode. This causes a leading and trailing edge shock wave which transform into expansion fans as the panel deflects downwards. The position and angle of the shock waves and expansion fans is found to be static. The flow field is completely attached, i.e. no buffet occurs. At high dynamic pressures (ref. to Fig. 14c and Fig. 14d) the inviscid and viscous solutions differ significantly due to the mode change at inviscid flow conditions. Figure 15 additionally shows the panel shape and the respective surface pressure distribution over one LCO period ( $\phi$ represents the phase angle). At $\phi=90^{\circ}$ and $\phi=270^{\circ}$ the existence of higher modes is recognizable. Shock waves and expansion fans are only observed at the leading and trailing edge and are stronger developed if a turbulent boundary layer is absent. This is an effect of shock-boundary layer interaction. Reduced flow velocities in the vicinity of the structure allow flow characteristics to propagate in flow direction as the local Mach number becomes lower than one and consequently pressure gradients are reduced. In the case of inviscid solutions flow discontinuities are transported from the trailing edge in streamwise direction. As observed for lower dynamic pressures the leading and trailing edge shock waves and expansion fans are still static in terms of position and angle. For dynamic pressures considered in this study no flow detachment occurs.

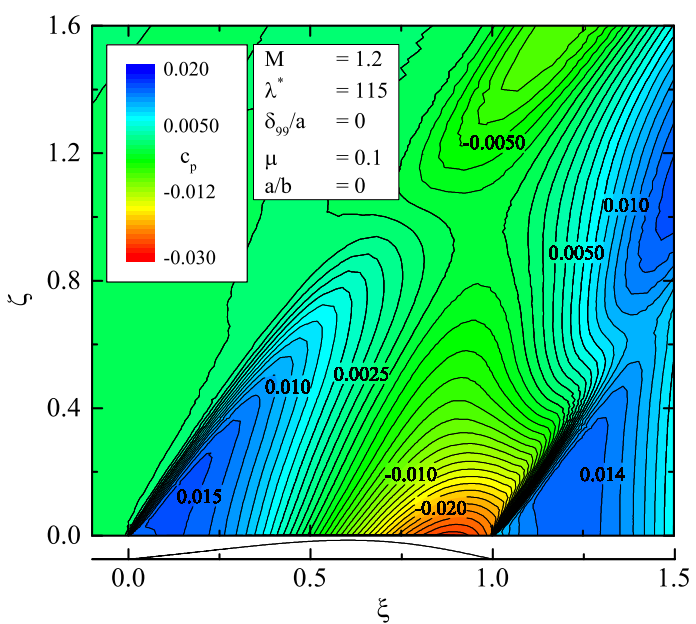

a) Inviscid (low dynamic pressure)

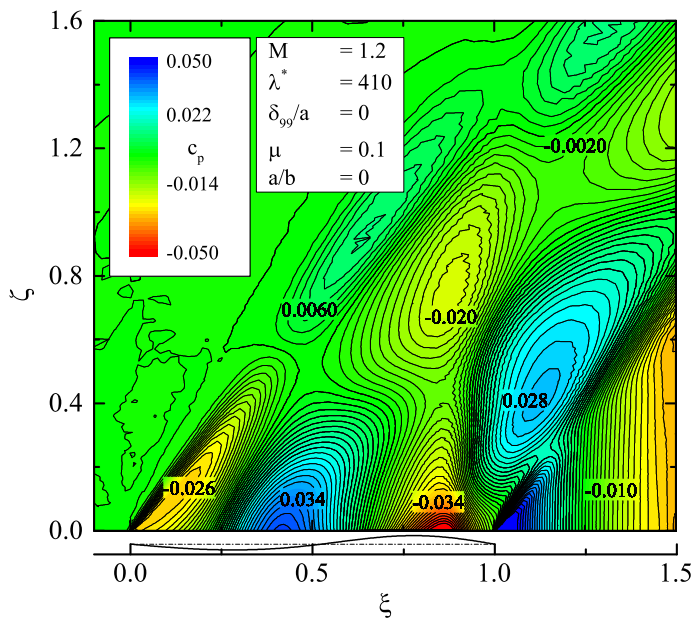

c) Inviscid (high dynamic pressure)

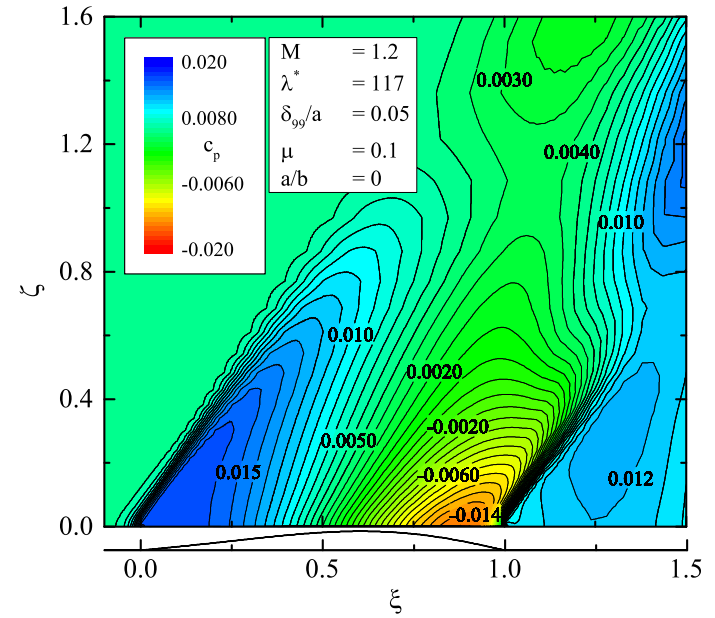

b) Viscous (low dynamic pressure)

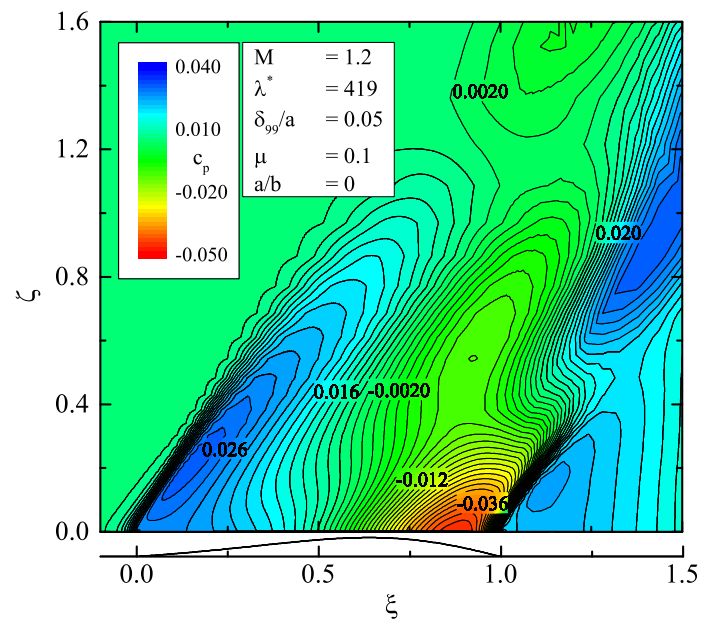

d) Viscous (high dynamic pressure)

Fig. 14. Pressure distribution at Mach 1.2 for inviscid (Euler) and viscous (SA) flow conditions. 


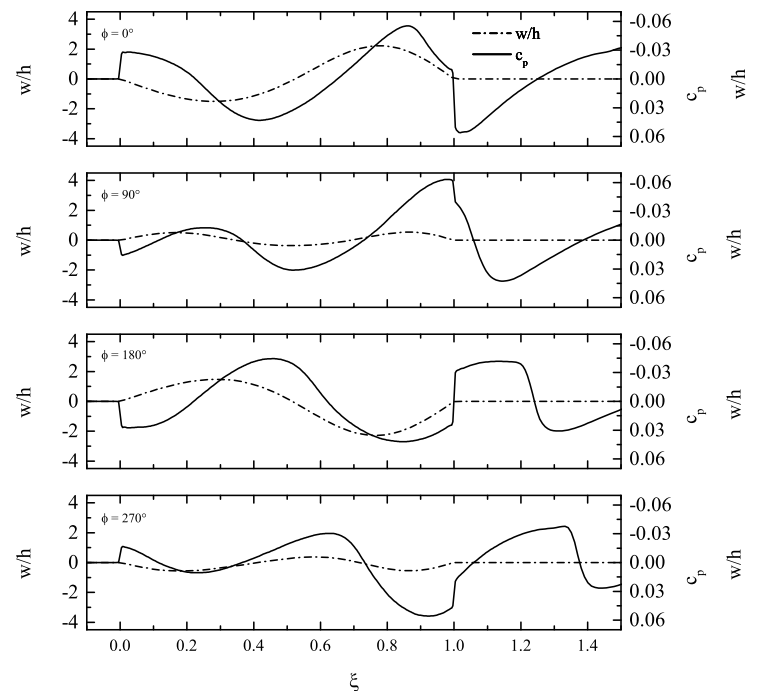

a) Inviscid flow $\left(\lambda^{*}=410\right)$
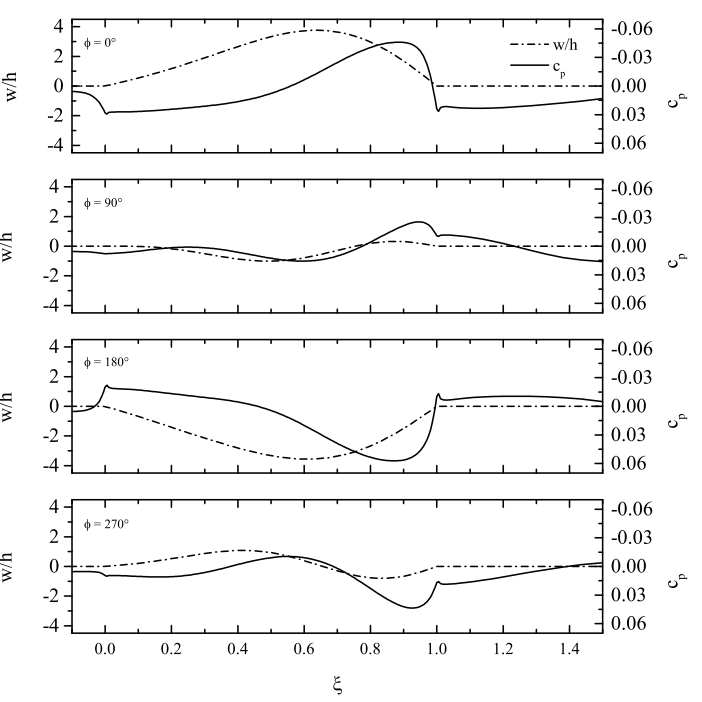

b) Viscous flow $\left(\lambda^{*}=419\right)$

Fig. 15. Panel displacement and surface pressure distribution for a LCO at Mach 1.2.

\section{Conclusion}

A partitioned solver for coupled 2D and 3D fluid-structure interaction has been developed for transonic panel flutter analysis. A high fidelity FEM solution as well as a Galerking solution of the von-Kármán plate equation has been coupled with URANS solutions to account for nonlinear unsteady aerodynamics. Within a detailed validation process for panels supported on all edges applicable results from literature as well as analytical models have been discussed in order to propose appropriate validation examples. In this framework it has been proven that, due to a subiteration strategy, the code is numerically stable and important physical effects such as static and dynamic instability are correctly reproduced. For the cases considered herein the applied initial conditions only affect static instabilities such as positive or negative divergence.

Based on the successful validation a parameter study has been conducted to study the impact of a turbulent boundary layer on aeroelastic stability and post-flutter characteristics of a $2 \mathrm{D}$ simply supported panel. A Mach number range from 0.85 to 2.0 has been considered. The following results were found:

(1) A turbulent boundary layer has a stabilizing effect on flat plates exposed to subsonic and low supersonic flow, i.e. the critical dynamic pressure is increased. As the Mach number increases the boundary layer has a considerable damping effect on higher modes involved in LCOs. At slightly higher Mach numbers (depending on geometry and boundary conditions approximately between Mach 1.5 and 1.7) the effect may be inverted and flutter onset occurs at higher dynamic pressures in the absence of a turbulent boundary layer. Exceeding Mach 1.6 the impact of a turbulent boundary layer becomes less significant and analytical aerodynamic models such as the quasi-static 'piston' theory become applicable.

(2) Since Hashimoto et al. ${ }^{11}$ also observe a negative damping effect of a turbulent boundary layer at Mach numbers between 1.3 and 1.8 for a 3D flat plate, it is shown that characteristic effects of viscous flows can be qualitatively reproduced by $2 \mathrm{D}$ computations. Although the sensitivity of semi-infinite plates on dynamic pressure and Mach number is much larger, this results is important for more detaild analysis of transonic panel flutter with respect to the computational effort of numerical experiments.

(3) The dependence of the critical dynamic pressure for flutter onset on the turbulent boundary layer thickness mainly shows a linear behavior solving URANS equations. This is as well observed experimentally by Muhlstein et al. ${ }^{26}$ and numerically by Dowell. ${ }^{28}$

(4) If flutter is a design criterion for space launcher structures, flow conditions in the vicinity of Mach 1.2 are crucial. Thus, this Mach number has been chosen for further analysis. As the dynamic pressure increases, higher flutter modes become more significant. A sudden mode change as observed for inviscid computations is considerably damped and possibly shifted to much larger dynamic pressures. This is in accordance with previous results on stability limits were higher flutter modes are also damped in the vicinity of the flutter 
boundary. Especially regarding aircraft flying at low supersonic Mach numbers this is important in terms of the fatigue behavior.

In this paper only an outline of a detailed analysis could be provided. Further work is necessary to study the phenomenology of the impact of viscous boundary layers on energy transfer between fluid and structure with respect to transonic panel flutter problems.

\section{Acknowledgments}

This study is conducted in cooperation with the DLR Institute of Aeroelasticity in Göttingen and Airbus DS in Bremen within the research cooperation Upper Stage and the PREPARE project. Sincere thank goes to Jannis Lübker (DLR) and Dr. Bernhard Kotzias (Airbus DS).

\section{References}

\footnotetext{
${ }^{1}$ Nichols, J., "Final report: Saturn V, S-IVB panel flutter qualification test," NASA Technical Note D-5439, 1969.

${ }^{2}$ Lall, T., "Interstage adapter panel flutter on Atlas-Centaur AC-2, AC-3, and AC-4 vehicles," NASA Technical Memorandum $X-1179,1965$.

${ }^{3}$ Dowell, E. H., Aeroelasticity of plates and shells, Noordhoff International, Leyden, 1975.

${ }^{4}$ Dowell, E. H., "Panel flutter - A review of the aeroelastic stability of plates and shells," AIAA Journal, Vol. 8, No. 3, 1970, pp. 385-399.

${ }^{5}$ Ashley, H. and Zartarian, G., "Piston Theory-A New Aerodynamic Tool for the Aeroelastician," Journal of the Aeronautical Sciences, Vol. 23, No. 12, 1956, pp. 1109-1118.

${ }^{6}$ Dowell, E. H., "Generalized aerodynamic forces on a flexible plate undergoing transient motion in a shear flow with an application to panel flutter," American Institute of Aeronautics and Astronautics, 1970.

${ }^{7}$ Muhlstein, L., Gaspers, P. A., and Riddle, D. W., "An experimental study of the influence of the turbulent boundary layer on panel flutter," NASA Technical Note D-4486, 1968.

${ }^{8}$ Mei, C., Abdel-Motagaly, K., and Chen, R., "Review of Nonlinear Panel Flutter at Supersonic and Hypersonic Speeds," Applied Mechanics Reviews, Vol. 52, No. 10, 1999, pp. 321.

${ }^{9}$ Davis, G. A. and Bendiksen, O. O., "Transonic panel flutter," AIAA Paper 93-1476, 1993.

${ }^{10}$ Gordnier, R. E. and Visbal, M. R., "Development of a three-dimensional viscous aeroelastic solver for nonlinear panel flutter," Journal of Fluids and Structures, Vol. 16, No. 4, 2002, pp. 497-527.

${ }^{11}$ Hashimoto, A., Aoyama, T., and Nakamura, Y., "Effects of Turbulent Boundary Layer on Panel Flutter," AIAA Journal, Vol. 47, No. 12, 2009, pp. 2785-2791.

${ }^{12}$ Felippa, C. A., Park, K., and Farhat, C., "Partitioned analysis of coupled mechanical systems," Computer Methods in Applied Mechanics and Engineering, Vol. 190, No. 24-25, 2001, pp. 3247-3270.

${ }^{13}$ Spalart, P. R. and Allmaras, S., "A one-equation turbulence model for aerodynamic flows," AIAA Paper 92-0439, 1992.

${ }^{14}$ Menter, F., "Zonal Two Equation k-w Turbulence Models For Aerodynamic Flows," AIAA Paper 93-2906, 1993.

${ }^{15}$ Schwamborn, D., Gerhold, T., and Heinrich, R., "The DLR TAU-Code: Recent Applications in Research and Industry," European Community on Computational Methods in Applied Sciences (ECCOMAS), 2006.

${ }^{16}$ Gerhold, T., Galle, M., Friedrich, O., and Evans, J., "Calculation of complex three-dimensional configurations employing the DLR-tau-code," 35th Aerospace Sciences Meeting and Exhibit, 1997.

${ }^{17}$ Reissner, E., "The effect of transverse shear deformation on the bending of elastic plates," ASME Journal of Applied Mechanics, Vol. 12, 1945.

${ }^{18}$ Mindlin, R., "Influence of rotatory inertia and shear on flexural motions of isotropic, elastic plates," ASME Journal of Applied Mechanics, Vol. 18, 1951, pp. 31-38.

${ }^{19}$ Dowell, E. H., "Nonlinear oscillations of a fluttering plate," AIAA Journal, Vol. 4, No. 7, 1966, pp. 1267-1275.

${ }^{20}$ Selvam, R., Visbal, M., and Morton, S., "Computation of nonlinear viscous panel flutter using a fully-implicit aeroelastic solver," 39th AIAA/ASME/ASCE/AHS/ASC Structures, 1998.

${ }^{21}$ Roache, P., "Perspective: A Method for Uniform Reporting of Grid Refinement Studies," Journal of Fluids Engineering, Vol. 116, 1994.

${ }^{22}$ Dugundji, J., Dowell, E. H., and Perkin, B., "Subsonic Flutter of Panels on Continous Elastic Foundations," AIAA Journal, Vol. 1, No. 5, 1963, pp. 1146-1154.

${ }^{23}$ Dowell, E. H., "Generalized Aerodynamic Forces on a Flexible Plate Undergoing Transient Motion," Quarterly of Applied Mathematics, Vol. 24, No. 4, 1967, pp. 331-338.

${ }^{24}$ Dowell, E. H., "Nonlinear oscillations of a fluttering plate. II," AIAA Journal, Vol. 5, No. 10, 1967, pp. $1856-1862$.

${ }^{25}$ Gaspers, P. A., Muhlstein, L., and Petroff, D. N., "Further experimental results on the influence of the turbulent boundary layer on panel flutter," NASA Technical Note D-5798, 1970.

${ }^{26}$ Muhlstein, Jr., L., "A forced-vibration technique for investigation of panel flutter," AIAA Paper 66-769, 1966.

${ }^{27}$ Braeunig, R., "Saturn V Launch Simulation," 2013, Retrieved May 26, 2014, from http://www.braeunig.us/apollo/ saturnV.htm.

${ }^{28}$ Dowell, E. H., "Aerodynamic Boundary Layer Effects on Flutter and Damping of Plates," AIAA Journal of Aircraft, Vol. 10, No. 12, 1973, pp. 734-738.
} 